\title{
Micro RNAs Versus Traditional Biomarkers in Predicting Reperfusion Injury in Hepatitis C Patients after Major Surgery
}

\author{
Hala Mourad Demerdash ${ }^{1 *}$, Emad Arida ${ }^{2}$, Ola Zanaty ${ }^{2}$ and Alaa Dawood ${ }^{2}$ \\ 'Pharos University in Alexandria, Alexandria University Hospitals, Egypt \\ ${ }^{2}$ Alexandria Faculty of Medicine, Egypt
}

\begin{abstract}
Background: Exposure to anesthesia in patients with chronic hepatitis C (CHC) may induce hepatic ischemia / reperfusion (I/R) with subsequent biochemical changes, provoked by alteration in molecular processes. This study explored miR-122 and miR-223 expression changes induced by anesthesia in CHC patients and their role in predicting hepatic I/R injury.

Methods: This study included two hundered ASA II - III adult patients, scheduled for major surgery under general anesthesia. Patients were divided into 2 main groups; CHC patients group and non-hepatitis patients group. Each group was further divided into 2 equal subgroups (50 patients each) according to type of anesthesia received either Sevoflurane SC /S or Propofol PC/P ). Blood samples were analyzed for liver enzymes, prothrombin time, and serum lactate at several intervals; preoperative, 12 and 48 hours postoperative. Also, plasma miR-122 and miR-223 were estimated by Reverse Transcription PCR preoperative and 12 hours postoperative.
\end{abstract}

Results: CHC groups showed significantly increased preoperative expression of both miR-122 and miR-223 compared to non-hepatitis groups $(\mathrm{P}<0.05)$. Moreover, postoperative samples revealed more exaggerated expression miR-122 and miR-223. Receiver operating characteristic curve (ROC) results revealed;values for area under the ROC for miR-122, miR-223, ALT and serum Lactate were 0.991, $0.965,0.746$ and 0.732 respectively. Based on the ROC finding, for prediction IR in CHC patients receiving anesthesia miR-122 had best Accuracy, sensitivity, and specificity.

Conclusion: Our results suggested that both miR-122 and miR-223 could be regarded as more sensitive and specific biomarkers for predicting hepatic I/R injury than traditional biomarkers particularly in CHC patients undergoing major surgery.

\section{Keywords: miRNA; Chronic Hepatitis C; Ischemia; Reperfusion; Propofol; Sevoflurane}

\section{Introduction}

Patients with Chronic Hepatitis C (CHC) are at predominantly high risk for morbidity and mortality as result of the effects of general anesthesia and stress of surgery. ${ }^{[1]}$ Since, the reciprocal flow relation between the hepatic artery and the portal vein is not well maintained, liver architecture is disrupted by fibrosis with consequent alteration of the normal patterns of liver blood flow which makes such patients more prone to ischemia/reperfusion $(\mathrm{I} / \mathrm{R}){ }^{[1,2]}$

Ischemia reperfusion (I/R) is defined as diminished oxygen supply to tissues to concentrations that are insufficient to maintain physiologic cell function. Reperfusion is essential for survival of ischemic tissue. ${ }^{[3]}$ However, reperfusion can injure ischemic tissue when the blood supply returns to the tissue after a period of ischemia. Since, absence of oxygen from blood during the ischemic period creates a condition in which the restoration of circulation results in inflammation and oxidative damage through release of cytokines from activated endothelium and tissue macrophages, endothelial adhesion/ dysfunction and migration of neutrophils. ${ }^{[3,4]}$
Exposure to anesthesia in $\mathrm{CHC}$ patients may aggrevate hepatic ischemia/reperfusion(I/R) with subsequent physiological and biochemical outcomes. These outcomes are somewhat mediated by alteration in molecular processes such as transcription and translation. The gold standard method to evaluate degree of liver damage after $\mathrm{I} / \mathrm{R}$ is by measuring serum aspartate aminotransferase (AST) and alanine . aminotransferase (ALT) levels and lactate dehydrogenase (LDH). However, interpretation of these enzymes levels may be difficult due to their existence in other tissues. ${ }^{[5]}$ MicroRNAs (miRNAs) are small noncoding RNAs that are implicated in regulation of messenger RNA degradation or translational repression. miRNAs negatively regulate more than $30 \%$ of the genes in cells, and control cell proliferation, inflammation, and metabolism. ${ }^{[6]}$ Moreover, miRNAs are extremely stable and are resistant to degradtion; Scince, plasma miRNAs are bound to highdensity lipoprotein and to Argonaute-proteins, which stabilizes them for long periods. ${ }^{[7]}$ A number of specific miRNAs are modulated in liver diseases; particularly, miR-122 is expressed in solely in hepatocytes, and hepatic 
cell death and necro-inflammatory activity in patients with CHC results in elevated expression of plasma miR-122. Also, experimental studies revealed change in expression of plasma miR-223 in I/R. ${ }^{[8]}$ However, the role of miR-223 in $\mathrm{I} / \mathrm{R}$ injury in humans has seldom been reported. ${ }^{[6]}$

Sevoflurane and propofol anesthesia are widely used for anesthesia and may influence hepatic blood flow, oxygen consumption and hepatic functions in the perioperative period, and they may influence the expression pattern of miRNA in CHC patients undergoing major surgery. ${ }^{[1]}$

This study investigated miR-122 and miR-223 expression changes induced by sevoflurane and propofol anesthesia in CHC patients and the role of these novel biomarkers in predicting hepatic injury during anesthesia versus traditional biomarkers.

\section{Patients and Methods}

The study was carried out in Alexandria Main University Hospital between January 2016 and January 2017. It was designed as double blind study, patients were assigned to groups at random. The study included 200 adult patients, ASA physical status II - III, scheduled for major surgeries with expected excessive blood loss ( othropedic surgery; spinal fixation, pelvic or compound femur fractures) under general anesthesia (GA). The research protocol was approved by the Ethics committee of the Faculty of Medicine, Alexandria University, Egypt. As well as in accordance with the ethical standards laid down in the 1964 declaration of Helsinki. Informed written consent was obtained from all participating patients.

Patients: All patients were subjected to full medical history, thorough clinical examination, laboratory investigation and ultrasound examination. They were divided into four groups; two groups were $\mathrm{CHC}$ patients and two groups were non-hepatitis patients.CHC parients were diagnosed by assays for HCV antibody testing, by using of a commercial recombinant immunoblot assay (Innolia HCV Ab III; Innogenetics) and confirmed by real-time quantitative HCV RNA PCR (more than $50 \mathrm{IU} / \mathrm{ml}$ ).

The Child-Pugh scoring system was performed for all CHC patients to assess the severity of cirrhosis, using grades for the following parameters of bilirubin, albumin, prothrombin time, hepatic encephalopathy and ascites from one to three to a maximum score of 15. [9] Non of patients in this study had Child-Pugh Class $\mathrm{C}$ cirrhosis. Exclusion criteria included pregnancy, malignancy, pulmonary or cardiovascular disease, concomitant Hepatitis B infection.

Studied patients were divided into two main groups ; CHC group and non-hepatitis group (one hundred patients each). Chronic hepatitis $\mathrm{C}(\mathrm{CHC})$ group (one hundred) were further divided into 2 subgroups according to anesthetic agent used; SC group (CHC patients who received Sevoflurane anesthesia ) and PC group (CHC patients who received propofol anesthesia), while non- hepatitis patients(one hundred) were divided into 2 subgroups: S group ( nonhepatitis patients who received Sevoflurane anesthesia ) and $\mathrm{P}$ group (non-hepatitis patients who received propofol anesthesia ). On arrival to operation room, patients were connected to (Trakmon-Kontron-Limited- England) multichannel monitor for routine monitoring and two large (16 gauge) intravenous cannulas were inserted.

Laboratory Investigations: Blood samples were collected pre-operative (before induction of anesthesia), 12 hours and 48 hours post-operative to determine the following tests:Serum samples for liver enzymes; serum aspartate aminotransferase (AST) and alanine aminotransferase (ALT), Blood samples were collected into Vacutainer tubes containing 0.129 M sodium citrate: prothrombin time (PT) and partial thromboplastin time (PTT) measured by a STA analyzer (DiagnosticaStago, Asnieres-sur-Seine, France), and EDTA blood for complete blood picture (CBC), determined by automated cell counter (ERMA INC-Tokyo. Model PCE-210). Also serum lactate levels were measured within 15-30 minutes using a point-of-care device, samples were kept on ice to avoid falsely elevated levels of lactate. In addition, blood samples were collected in EDTA tubes, centrifuged at $4000 \mathrm{rpm}$ for $10 \mathrm{~min}$ at $4{ }^{\circ} \mathrm{C}$, plasma was separated and stored at $-70^{\circ} \mathrm{C}$ for analysis of miR-122 and miR-223, samples were withdrawn pre-operatively and 12 hours post-operatively.

\section{miRNA Quantification by Real-Time PCR (RT-qPCR)}

RNA extraction and cDNA synthesis: Total RNA was extracted from $200 \mu \mathrm{l}$ plasma using the miRNeasy extraction kit (Qiagen, Valencia, CA, USA). RNA purity was assessed by the RNA concentration and quantified by NanoDrop Spectrophotometer (Nanodrop ND-1000, United States). Followed by analysis using agarose gel electrophoresis. Reverse transcription was performed for cDNA synthesis using the miRNeasy plasma Reverse Transcription Kit (Qiagen, Valencia, CA, USA) according to the manufacturer's instructions. Amplification and quantification

The expression miR-122 and miR-223 were evaluated by RT-qPCR analysis according to the manufacturer's protocol. The housekeeping miRNA SNORD 68 was used as the endogenous control. For real-time PCR, the cDNA template was mixed with SYBER Green Master Mix (Qiagen, Valencia, CA, USA). PCR array plate enriched with forward and reverse miRNA-specific primers. 
Real-time PCR reactions were performed using an Applied Biosystems 7500 Real Time PCR System (Foster city, CA, USA) with the following conditions: $95^{\circ} \mathrm{C}$ for $15 \mathrm{~min}$, followed by 40 cycles at $94^{\circ} \mathrm{C}$ for $15 \mathrm{~s}, 55^{\circ} \mathrm{C}$ for $30 \mathrm{~s}$ and $70^{\circ} \mathrm{C}$ for $34 \mathrm{~s}$. The cycle threshold (CT) is defined as the number of cycles required for the fluorescent signal to cross the threshold in real-time PCR.

Expression of miRNAs was reported as $\Delta \mathrm{Ct}$ value. The $\Delta \mathrm{Ct}$ was calculated by subtracting the $\mathrm{Ct}$ values of miRNA SNORD68 from the $\mathrm{Ct}$ values of the target miRNAs. there is an inverse correlation between $\Delta \mathrm{Ct}$ and miRNA expression level, lower the $\Delta \mathrm{Ct}$ values were associated with increased miRNA. The resultant normalized $\Delta \mathrm{Ct}$ values were used in calculating relative expression values by using $2-\Delta(\mathrm{Ct})$, these values are directly related to the miRNA expression levels. The $2-\Delta \Delta(\mathrm{Ct})$ method was used to determine relative-quantitative levels of individual miRNAs.

Anesthetic Protocol: All patients were subjected to the same anesthetic protocol for induction of GA, using intravenous (IV) propofol $2-3 \mathrm{mg} / \mathrm{kg}$ and IV fentanyl $2 \mu \mathrm{g} / \mathrm{kg}$; endotracheal intubation was facilitated by IV atracurium $0.5 \mathrm{mg} / \mathrm{kg}$. Anesthesia in Groups S and SC patients were maintained by 2-3\% sevoflurane in oxygen and atracurium increments, while anesthesia in Groups $\mathrm{P}$ and $\mathrm{PC}$ patients were maintained by propofol infusion at a rate of $6 \mathrm{mg} / \mathrm{kg} / \mathrm{hr}$ and IV atracurium $0.5 \mathrm{mg} / \mathrm{kg}$ increments. In addition to standard monitoring [Heart rate (HR), Mean arterial blood pressure (MAP) and Pulse oxygen saturation $\left(\mathrm{SpO}_{2}\right)$ ] urinary output was recorded. At the end of surgery, residual neuromuscular block was antagonized with IV neostigmine $0.05 \mathrm{mg} / \mathrm{kg}$ and atropine $0.02 \mathrm{mg} / \mathrm{kg}$.

Fluid therapy was managed by administration of crystalloids and colloids solutions. Adequate replacement and maintenance of intravascular volume were guided by monitoring MAP, urinary output $(\geq 1 \mathrm{ml} / \mathrm{kg} / \mathrm{h}$ ) and hourly hematocrit measurement. Packed cells (PEs) was transfused when the hemoglobin decreased to less than $10 \mathrm{~g} / \mathrm{dl}$, to prevent inadequate oxygenation during rapid bleeding. Other blood products, such as fresh frozen plasma (FFP) and platelets were administered intraoperative in accordance with the recommendations of the ASA Task Force on Blood Component Therapy. [10] Operative time was recorded in minutes. Estimated blood volume loss during surgery was determined from the surgical suction reservoir and by weighing towels from the operative field; total intraoperative blood transfusion was calculated.

Statistical Analysis: The raw data were coded, entered, and analyzed using IBM SPSS Software Package version 20, The 0.05 level was used as the cut off value for statistical significance and the following statistical measures were used: Descriptive statistics including counts and percentages, Arithmetic mean, standard deviation, for categorized parameters, chai square test was used while for numerical data t-test was used to compare two groups while for more than two groups ANOVA test was used, followed by post hoc test to determine the level of significant between each two groups, it was done Duncan method, by using the small letters, the same letters indicate that there was no significant difference, while the differ small letters show a significant difference. To find the association between two variables, Spearman correlation coefficient test was used, the level of significant was 0.05 .

\section{Results}

This study included two hundred adult patients who underwent major orthropedic surgery with blood loss at Alexandria University hospital during study period. Patients were randomly divided into four equal groups (50 each); 2 CHC groups (SC and PC ), and 2 non- hepatitis groups ( $\mathrm{S}$ and $\mathrm{P}$ ). $\mathrm{CHC}$ patients included in study were classified according to Child-Pugh scoring ; SC group (CHC patients who received Sevoflurane) 14 patients were class A and 36 patients were class $\mathrm{B}$, while $\mathrm{PC}$ group $(\mathrm{CHC}$ patients who received propofol) 10 patients were class $\mathrm{A}$ and 40 patients were class B.

No significant differences were detected as regards age, gender, surgical time, urine output, blood loss and body temperature. None of the non-hepatitis groups ( $\mathrm{S}$ and $\mathrm{P}$ ) received blood transfusion, while 7 patients in SC group received 2 units of packed red blood cells each, while 2 patients in PC group received 2 units of packed red blood cells each. (Table 1)

MAP measures were significantly lower in both groups that received sevoflurane anesthesia (SC and $\mathrm{S}$ ) than groups that reveived propofol anesthesia ( $\mathrm{PC}$ and $\mathrm{P}$ ) at the end of surgery, with a more significant decrease in SC group compared to other groups. (Table 2) Platelets count was significantly lower in CHC groups (SC and $\mathrm{PC}$ ) than nonhepatitis groups ( $\mathrm{S}$ and $\mathrm{P}$ ); pre-operative, $12 \mathrm{hrs}$ and $48 \mathrm{hrs}$ post-operative. (Table 3)

PT values were significantly prolonged in both hepatitis groups (SC and PC) compared to non-hepatitis groups (S and P); Preoperative $(\mathrm{p} 1=0.012$ and $\mathrm{p} 2=0.011), 12$ hours post-operative $(\mathrm{p} 1=0.033$ and $\mathrm{p} 2=0.021)$ and 48 hours post-operative $(\mathrm{p} 1=0.003$ and $\mathrm{p} 2=0.015)$. with a significantly higher \% change in CHC group SC group compared to PC group $(\mathrm{P} \leq 0.05)$. Also, Serum AST levels were significantly elevated in hepatitis groups (SC and PC) compared to non- hepatitis groups (S and $\mathrm{P}$ ); Preoperative $(\mathrm{p} 1=0.002$ and $\mathrm{p} 2=0.001), 12$ hours post-operative $(\mathrm{p} 1=$ 
0.001 and $\mathrm{p} 2=0.001)$ and 48 hours post-operative ( $\mathrm{p} 1=$ 0.001 and $\mathrm{p} 2=0.001$ ) with a significantly higher $\%$ change in $\mathrm{CHC}$ group $\mathrm{SC}$ compared to $\mathrm{PC}$ group $(\mathrm{P} \leq 0.05)$. (Table 3)

Preoperative plasma expression miR-122 and miR-223 were significantly increased in both $\mathrm{CHC}$ groups (SC and $\mathrm{PC}$ ) compared to non-hepatitis groups ( $\mathrm{S}$ and $\mathrm{P}$ ). Post-operative samples ( after12 hours ) plasma miR-122 showed significantly increased expression in both $\mathrm{CHC}$ groups compared to non-hepatitis groups. Moreover, SC group revealed more significant increased expression compared to other groups. Similarly postoperative plasma miR-223 revealed more significant expression non-hepatitis group $\mathrm{S}$ (receiving Sevoflurane) relative group P (receiving Propofol). Also postoperative analysis revealed more significant increase in $\mathrm{CHC}$ groups with more pronounced expression in $\mathrm{SC}$ group compared to $\mathrm{PC}$ group. (Table 4)

Analysis of plasma miR-122 displayed a significant increase in CHC patients SC group (receiving Sevoflurane) with child pugh $\mathrm{B}$ both preoperative $(\mathrm{P}: 0.042)$ and 12 hours postoperative(P: 0.024). Also, plasma miR-233 was significantly increased in patients with child pugh B both preoperative (P: 0.011) and 12 hours postoperative (P: 0.016) in CHC patients in SC group compared to PC group. In the PC group miR-122 preoperative (P : 0.069) and 12 hours postoperative was (P: 0.077); while miR-223 preoperative (P : 0.032) and 12 hours postoperative was (P: 0.036).

ROC curve was designed for predicting hepatic IR in CHC patients receiving anesthesia, results revealed that miR122 had $97 \%$ sensitivity, 92\% specificity and 95\% accuracy (Figure1, Table 5)

Table 1: Demographic \& Physiological Data for all studied groups.

\begin{tabular}{|c|c|c|c|c|}
\hline & SC & $\mathrm{S}$ & PC & $\mathbf{P}$ \\
\hline Age & $47.09 \pm 6.23$ & $44.45 \pm 7.78$ & $49.67 \pm 5.56$ & $46.23 \pm 7.10$ \\
\hline $\mathrm{P} 1$ & \multicolumn{2}{|c|}{0.213} & \multicolumn{2}{|c|}{0.336} \\
\hline $\mathrm{P} 2$ & & & 0.112 & 0.207 \\
\hline Gender (M/F) & $(12 / 8)$ & $(10 / 10)$ & $(12 / 8)$ & $(11 / 9)$ \\
\hline $\mathrm{P} 1$ & \multicolumn{2}{|c|}{0.425} & \multicolumn{2}{|c|}{0.365} \\
\hline $\mathrm{P} 2$ & & & 1.00 & 0.854 \\
\hline Duration of operation(min) & $139 \pm 18$ & $132 \pm 13$ & $146 \pm 19$ & $139 \pm 21$ \\
\hline $\mathrm{P} 1$ & \multicolumn{2}{|c|}{0.236} & \multicolumn{2}{|c|}{0.411} \\
\hline $\mathrm{P} 2$ & & & 0.078 & 0.365 \\
\hline Blood loss $(\mathrm{ml})$ & $1078 \pm 271$ & $916 \pm 129$ & $1012 \pm 254$ & $930 \pm 168$ \\
\hline P1 & \multicolumn{2}{|c|}{0.107} & \multicolumn{2}{|c|}{0.116} \\
\hline $\mathrm{P} 2$ & & & 0.689 & 0.722 \\
\hline Volume of fluid infused(ml) & $1800 \pm 420$ & $1680 \pm 510$ & $2000 \pm 495$ & $1780 \pm 470$ \\
\hline $\mathrm{P} 1$ & \multicolumn{2}{|c|}{$0.031^{*}$} & \multicolumn{2}{|c|}{$0.041^{*}$} \\
\hline $\mathrm{P} 2$ & & & 0.101 & 0.223 \\
\hline Urine output & $355 \pm 165$ & $368 \pm 159$ & $386 \pm 145$ & $377 \pm 148$ \\
\hline $\mathrm{P} 1$ & \multicolumn{2}{|c|}{0.652} & \multicolumn{2}{|c|}{0.482} \\
\hline $\mathrm{P} 2$ & & & 0.136 & 0.254 \\
\hline Temperature at the end of surgery $\left({ }^{\circ} \mathrm{C}\right)$ & $36.0 \pm 0.4$ & $36.5 \pm 0.4$ & $35.9 \pm 0.3$ & $36.7 \pm 0.5$ \\
\hline $\mathrm{P} 1$ & \multicolumn{2}{|c|}{0.682} & \multicolumn{2}{|c|}{0.211} \\
\hline P2 & & & 0.365 & 0.852 \\
\hline
\end{tabular}

* Statistically significant at $(\mathrm{P} \leq 0.05)$

SC: CHC patients who received Sevoflurane, S: non-hepatitis patients who received Sevoflurane, PC: CHC patients who received Propofol, P: non-hepatitis patients who received Propofol.

P1: between groups receiving same anesthetic drug.

P2 : between 2 control groups and 2 hepatitis groups. 
Table 2: Hemodynamic measurements for all studied groups during surgery.

\begin{tabular}{|c|c|c|c|c|}
\hline & SC & S & PC & $\mathbf{P}$ \\
\hline \multicolumn{5}{|c|}{ Mean arterial blood pressure $(\mathrm{mmHg})$ : } \\
\hline *Preoperative & $94 \pm 21$ & $93 \pm 17$ & $103 \pm 15$ & $98 \pm 20$ \\
\hline P1 & \multicolumn{2}{|c|}{0.311} & \multicolumn{2}{|c|}{0.207} \\
\hline $\mathrm{P} 2$ & & & $0.032^{*}$ & $0.041^{*}$ \\
\hline $\begin{array}{l}{ }^{*} \text { Intra-operative (30 min after } \\
\text { start of surgery) }\end{array}$ & $88 \pm 14$ & $90 \pm 13$ & $102 \pm 17$ & $96 \pm 18$ \\
\hline P1 & \multicolumn{2}{|c|}{0.103} & \multicolumn{2}{|c|}{0.412} \\
\hline $\mathrm{P} 2$ & & & $0.001^{*}$ & $0.046^{*}$ \\
\hline${ }^{*}$ At the end of surgery & $84 \pm 20$ & $90 \pm 18$ & $104 \pm 17$ & $94 \pm 16$ \\
\hline P1 & \multicolumn{2}{|c|}{$0.041^{*}$} & \multicolumn{2}{|c|}{0.106} \\
\hline P2 & & & $0.002^{*}$ & 0.107 \\
\hline \multicolumn{5}{|l|}{ Heart rate (beat per min): } \\
\hline${ }^{*}$ Preoperative & $75 \pm 15$ & $78 \pm 14$ & $67 \pm 12$ & $73 \pm 14$ \\
\hline P1 & \multicolumn{2}{|c|}{0.365} & \multicolumn{2}{|c|}{0.085} \\
\hline $\mathrm{P} 2$ & & & $0.017^{*}$ & 0.165 \\
\hline $\begin{array}{l}{ }^{*} \text { Intra-operative } \\
\text { (30 min after start of surgery) }\end{array}$ & $72 \pm 10$ & $77 \pm 15$ & $70 \pm 13$ & $75 \pm 12$ \\
\hline P1 & \multicolumn{2}{|c|}{0.071} & \multicolumn{2}{|c|}{0.103} \\
\hline P2 & & & 0.235 & 0.365 \\
\hline${ }^{*} \mathrm{At}$ the end of surgery & $71 \pm 14$ & $74 \pm 16$ & $67 \pm 17$ & $73 \pm 15$ \\
\hline P1 & \multicolumn{2}{|c|}{0.336} & \multicolumn{2}{|c|}{0.144} \\
\hline $\mathrm{P} 2$ & & & 0.185 & 0.236 \\
\hline
\end{tabular}

* Statistically significant at $(\mathrm{P} \leq 0.05)$

$\mathrm{SC}$ : CHC patients who received Sevoflurane, S: non-hepatitis patients who received Sevoflurane. While PC: CHC patients who received Propofol, P: non-hepatitis patients who received Propofol.

$P 1$ : between groups receiving same anesthetic drug.

$P 2$ : between 2 control groups and 2 hepatitis groups.

Table 3: laboratory investigations measured preoperative, postoperative after 12 hours and 48 hours in all studied groups.

\begin{tabular}{|c|c|c|c|c|c|c|c|}
\hline & SC & $\mathrm{s}$ & PC & $\mathbf{P}$ & P 1 & P 2 & P3 \\
\hline \multicolumn{8}{|l|}{ ALT (IU/ml): } \\
\hline *Preoperative & $47.43 \pm 12.45$ & $28.67 \pm 14.23$ & $62.87 \pm 15.88$ & $23.45 \pm 11.89$ & $0.022^{*}$ & $0.001^{*}$ & $0.016^{*}$ \\
\hline *12 hrs post-op. & $110.68 \pm 41.77$ & $44.23 \pm 19.65$ & $112.78 \pm 72.69$ & $45.43 \pm 13.89$ & $0.003^{*}$ & $0.001^{*}$ & 0.211 \\
\hline *48 hrs post-op. & $398.32 \pm 56.56$ & $36.41 \pm 17.13$ & $291.37 \pm 61.38$ & $38.26 \pm 18.67$ & $0.001^{*}$ & $0.001^{*}$ & $0.017^{*}$ \\
\hline \multicolumn{8}{|c|}{ Hemoglobin (gm/dl): } \\
\hline *Preoperative & $11.6 \pm 2.8$ & $14.1 \pm 1.6$ & $11.3 \pm 2.1$ & $14.1 \pm 0.7$ & $0.039^{*}$ & $0.041^{*}$ & 0.699 \\
\hline *12 hrs post-op. & $10.2 \pm 1.6$ & $13.9 \pm 1.4$ & $11.0 \pm 0.8$ & $13.9 \pm 1.8$ & $0.036^{*}$ & 0.068 & 0.356 \\
\hline *48 hrs post-op. & $10.1 \pm 1.8$ & $13.9 \pm 1.56$ & $10.9 \pm 0.9$ & $14.0 \pm 0.92$ & $0.035^{*}$ & $0.041^{*}$ & 0.32 \\
\hline \multicolumn{8}{|c|}{ Serum lactate $(\mathrm{mmol} / \mathrm{L})$} \\
\hline *Preoperative & $1.96 \pm 0.48$ & $1.21 \pm 0.34$ & $1.82 \pm 0.43$ & $1.01 \pm 0.31$ & $0.033^{*}$ & $0.041^{*}$ & 0.365 \\
\hline *12 hrs post-op. & $5.67 \pm 1.98$ & $2.66 \pm 1.78$ & $4.54 \pm 1.88$ & $2.83 \pm 1.91$ & $0.003^{*}$ & $0.0021^{*}$ & 0.106 \\
\hline *48 hrs post-op. & $3.23 \pm 1.77$ & $1.85 \pm 1.04$ & $2.25 \pm 1.52$ & $1.71 \pm 1.1$ & $0.026^{*}$ & $0.041^{*}$ & 0.107 \\
\hline
\end{tabular}

* Statistically significant at $(\mathrm{P} \leq 0.05)$

SC: CHC patients who received Sevoflurane, S: non-hepatitis patients who received Sevoflurane, PC: CHC patients who received Propofol, P: non-hepatitis patients who received Propofol.

$\mathrm{P} 1$ comparison between $\mathrm{S}$ and $\mathrm{SC}$.

$\mathrm{P} 2$ comparison between $\mathrm{P}$ and $\mathrm{PC}$.

$\mathrm{P} 3$ comparison between $\mathrm{SC}$ and $\mathrm{PC}$. 
Table 4: Plasma miR-122 and miR-223 expression preoperative and 12 hours postoperative in all studied groups.

\begin{tabular}{|c|c|c|c|c|c|c|c|}
\hline \multicolumn{8}{|c|}{ Plasma miR-122 in all studied groups } \\
\hline \multicolumn{3}{|c|}{ Sevoflurane Anesthesia Groups } & \multirow[t]{2}{*}{ P1 } & \multicolumn{3}{|c|}{ Propofol Anesthesia Groups } & \multirow[t]{2}{*}{ P1 } \\
\hline & Preoperative & 12 hrs Postoperative & & & Preoperative & $\begin{array}{c}12 \mathrm{hrs} \\
\text { Postoperative }\end{array}$ & \\
\hline$S$ & $0.78 \pm 0.31$ & $0.99 \pm 0.18$ & 0.107 & $\mathrm{P}$ & $0.72 \pm 0.27$ & $0.96 \pm 0.23$ & 0.211 \\
\hline SC & $7.02 \pm 1.77$ & $8.92 \pm 1.07$ & 0.203 & PC & $6.52 \pm 0.92$ & $7.46 \pm 1.03$ & 0.071 \\
\hline $\mathrm{P} 2$ & $0.001^{*}$ & $0.001^{*}$ & & & $0.001^{*}$ & $0.001^{*}$ & \\
\hline \multicolumn{8}{|c|}{ plasma miR-223 in all studied groups } \\
\hline \multicolumn{3}{|c|}{ Sevoflurane Anesthesia Groups } & $\mathrm{P} 1$ & & \multicolumn{2}{|c|}{ Propofol Anesthesia Groups } & $\mathrm{P} 1$ \\
\hline & Preoperative & 12 hrs Postoperative & & & Preoperative & $\begin{array}{c}12 \text { hrs } \\
\text { Postoperative }\end{array}$ & \\
\hline $\mathrm{S}$ & $0.811 \pm 0.041$ & $1.02 \pm 0.23$ & $0.032^{*}$ & $\mathrm{P}$ & $0.726 \pm 0.09$ & $0.83 \pm 0.03$ & 0.425 \\
\hline SC & $4.21 \pm 0.33$ & $6.07 \pm 1.07$ & $0.013^{*}$ & $\mathrm{PC}$ & $3.96 \pm 0.285$ & $5.33 \pm 0.18$ & $0.021^{*}$ \\
\hline $\mathrm{P} 2$ & $0.001^{*}$ & $0.001^{*}$ & & & $0.001^{*}$ & $0.001^{*}$ & \\
\hline
\end{tabular}

* Statistically significant at $(\mathrm{P} \leq 0.05)$

SC: CHC patients who received Sevoflurane, S: non-hepatitis patients who received Sevoflurane, PC: CHC patients who received Propofol, P: non-hepatitis patients who received Propofol.

$\mathrm{P} 1$ comparison between pre and post in the same group

P2 comparison between the two groups at the same time

Table 5: Cut off value of different biomarkers evaluated to predict the IR injury in CHC patients undergoing major surgery.

\begin{tabular}{|c|c|c|c|c|c|c|c|c|}
\hline \multirow[t]{2}{*}{$\begin{array}{l}\text { Test Result } \\
\text { Variable(s) }\end{array}$} & \multirow[t]{2}{*}{$\begin{array}{l}\text { Area under } \\
\text { the curve }\end{array}$} & \multirow[t]{2}{*}{$p$-value } & \multirow[t]{2}{*}{$\begin{array}{l}\text { Cut off } \\
\text { value }\end{array}$} & \multirow[t]{2}{*}{ Sensitivity } & \multirow[t]{2}{*}{ Specificity } & \multirow[t]{2}{*}{ Accuracy } & \multicolumn{2}{|c|}{$\begin{array}{l}\text { Asymptotic } 95 \% \\
\text { Confidence Interval }\end{array}$} \\
\hline & & & & & & & $\begin{array}{l}\text { Lower } \\
\text { Bound }\end{array}$ & $\begin{array}{l}\text { Upper } \\
\text { Bound }\end{array}$ \\
\hline ALT & .746 & .012 & 32.0 & 75.0 & 72.0 & 74.0 & .676 & .815 \\
\hline Serum lactate & .732 & .011 & 1.42 & 70.0 & 74.0 & 72.0 & .661 & .803 \\
\hline miR-122 & .991 & .0001 & 0.68 & 97.0 & 92.0 & 95.0 & .976 & 1.000 \\
\hline miR-223 & .965 & .0001 & 0.75 & 95.0 & 90.0 & 92.0 & .944 & .987 \\
\hline
\end{tabular}

Table 5: Cut off value of different biomarkers evaluated to predict the IR injury in CHC patients undergoing major surgery.

\begin{tabular}{|c|c|c|c|c|c|c|c|c|}
\hline \multirow[t]{2}{*}{$\begin{array}{l}\text { Test Result } \\
\text { Variable(s) }\end{array}$} & \multirow[t]{2}{*}{$\begin{array}{l}\text { Area under } \\
\text { the curve }\end{array}$} & \multirow[t]{2}{*}{ p-value } & \multirow[t]{2}{*}{$\begin{array}{l}\text { Cut off } \\
\text { value }\end{array}$} & \multirow[t]{2}{*}{ Sensitivity } & \multirow[t]{2}{*}{ Specificity } & \multirow[t]{2}{*}{ Accuracy } & \multicolumn{2}{|c|}{$\begin{array}{l}\text { Asymptotic } 95 \% \\
\text { Confidence Interval }\end{array}$} \\
\hline & & & & & & & $\begin{array}{l}\text { Lower } \\
\text { Bound }\end{array}$ & $\begin{array}{l}\text { Upper } \\
\text { Bound }\end{array}$ \\
\hline ALT & .746 & .012 & 32.0 & 75.0 & 72.0 & 74.0 & .676 & .815 \\
\hline Serum lactate & .732 & .011 & 1.42 & 70.0 & 74.0 & 72.0 & .661 & .803 \\
\hline miR-122 & .991 & .0001 & 0.68 & 97.0 & 92.0 & 95.0 & .976 & 1.000 \\
\hline miR-223 & .965 & .0001 & 0.75 & 95.0 & 90.0 & 92.0 & .944 & .987 \\
\hline
\end{tabular}

\section{Discussion}

Abundant evidence revealed the involvement of miRNAs in different forms of liver disease; detailed mechanisms of the influence of anesthetic agents on the liver miRNAs expression during $\mathrm{I} / \mathrm{R}$ are still unknown. the two widely used general anesthetics propofol and sevoflurane, are known to influence hepatic cell function during the perioperative period. [13] In the present study, it was investigated whether plamsa miR-122 and miR-223 could serve as potential indicators of IR injury in CHC patients. Therefore, plasma expression levels of these miRNAs both preoperative and postoperative in patients undergoing 
major surgery with expected $\mathrm{I} / \mathrm{R}$ and were tested to determine whether their expression levels correlated with established enzyme of hepatocellular injury AST and ALT, as well as serum lactate levels.

Results of the current study showed elevated expression of plasma miR-122 in CHC groups (SC and PC) compared to non-hepatitis groups ( $\mathrm{S}$ and $\mathrm{P}$ ) in preoperative period. This was explained by the fact that miR-122 constitutes more than $70 \%$ of the total liver miRNA, and are released into circulation as result of hepatocyte injury due to $\mathrm{HCV}$ infection. Also, more exaggerated expression of plasma miR122 in $\mathrm{CHC}$ groups in the postoperative period compared to non-hepatitis groups, with more prominently increased expression in CHC group who received Sevoflurane (SC). Exaggerated expression of plasma miR-122 was explained by further release into the blood stream as a result of hepatic cell insult due to hypoperfusion, inflammatory condition and/or drug induced liver injury. [14,15] In addition, plasma miR-122 levels were found to be significantly associated with liver function parameters; ALT, PT and serum lactate particularly in CHC groups. Similarly, other studies reported that circulating level of miR-122 correlates with severity of hepatocyte injury. [16,17]

Hepatic injury as result of $I / R$ ensue from sinusoidal damage with subsequent microcirculatory disturbance upon reperfusion, resulting from prolonged hypotension and increased blood loss following hepatocyte injury. [18] Those outcomes were demonstrated by evaluation of liver function tests, serum lactate which reflect hepatocyte integrity. [15]

In present study liver enzymes; serum AST or ALT levels did not change significantly in 12 hours and 48 hours postoperative in both non-hepatitis groups ( $\mathrm{S}$ and $\mathrm{P}$ ). However, significantly elevated liver enzyme ALT and prolonged PT were observed in samples collected 12 and 48 hours post-operative in $\mathrm{CHC}$ groups with more significant increase in Sevoflurane group (SC). Those results determined presence of hepatic dysfunction in $\mathrm{CHC}$ patients with anesthesia particularly Sevoflurane, which was in agreement with results of Subhi et al study. [18] In addition, Choi et al reported that miR-122 regulates the levels of ALT without changing the transcriptional level of the coding gene, proposing that it is implicated in translation activation of ALT. [19]

Likewise, other studies confirmed that circulating miR-122 is proposed an early disease severity-dependent biomarker of liver injury as its levels become raised earlier than that of serum ALT. $[18,19,20]$ Moreover, significant increase in liver enzymes in postoperative periods (after 12 and 48 hours) in CHC groups. Hepatic enzyme ALT is involved in cellular nitrogen metabolism and gluconeogenesis by catalysing the transamination between alanine and 2-oxoglutarate, the preferential localisation of miR-122 around lipid droplets of the hepatocytes reinforces this perception. [21,22] Also, a novel role of miR-122 in the modulation of hepatic glucose metabolism and elucidated its profound relation with ALT. [20]

In addition, other studies stated that plasma miR-122 can be detected before histopathologic changes evolve in experimental models of cytotoxic liver-induced damage, and they agreed to consider miR-122 not only as a biomarker of hepatocellular necrosis, but also hepatocellular stress. $[6,20,23,24]$

Furthermore, sevoflurane is known to produce extra metabolite, Compound A [fluoromethyl-2,2-difluoro-1(difluoromethyl) ether], through chemical reactions with $\mathrm{CO}_{2}$ absorbents. [18] The resultant Compound A might be hepatotoxic as proved by transient increase in postoperative liver function tests as well as elevated plasma miR-122, miR-223 expression in our study. Also, a study performed on morbidly obese subjects undergoing surgery, reported that ALT has less specificity than miR-122 for identifying liver cell injury because of its presence in the heart and skeletal muscle. [17]

Moreover, significantly elevated serum lactate in $\mathrm{CHC}$ groups after 12 and 48 hours post-operative was the result of impaired lactate clearance due cellular hypoperfusion or hypoxia and anaerobic metabolism. [25]

As regard plasma miR-223; it functions as an important modulator of cellular differentiation, including dendritic cells and macrophage activation. [26] Furthermore, miR-223 influences the inflammatory response through activation of neutrophils and the secretion of inflammatory cytokines. [27] Furthermore, miR-223 in the liver imparts a potential role in modulation of the pharmacokinetics. This regulatory mechanism might be a supplementary factor for the inter- and intra-individual variability in drugs pharmacokinetics. Cytochrome b5 (b5) is posttranscriptionally regulated through miR-223. The b5 is a hemoprotein that transfers electrons to several enzymes to accomplish fatty acid desaturation, steroidogenesis, and drug metabolism. [28]

Hepatocyte damage in $\mathrm{I} / \mathrm{R}$ is partially due oxygen-derived free radicals due to lipid peroxidation, especially during reperfusion. cytochrome b5 increases during ischemic periods, followed by immense increase $60 \mathrm{~min}$ after reperfusion. it exerts a protective effect against liver cell damage, such as free radical-trapping ability and or membrane stabilizing action. [28,29] 
Pronounced expression of postoperative plasma miR-223 in $\mathrm{CHC}$ group (SC); could be explained by presence of additional mechanism of $\mathrm{I} / \mathrm{R}$ includes damage caused by sustained accumulation of leukocytes; with consequent obstruction of microcirculation, release of non-enzymatic components for a prolonged period after their activation; furthermore produced cytokines might result in hepatocyte injury. [30,31] miR-223 might be useful as an indicator of liver I/R injury as it expression is elevated and was positively correlated with ALT level and elevated serum lactate 12 hours postoperative in both $\mathrm{CHC}$ groups (SC and PC). Accordingly, $\mathrm{Yu}$ et al suggested that miR-223 is a more sensitive indicator of hepatic $\mathrm{I} / \mathrm{R}$ injury than traditional liver function tests (ALT and AST).[26]

Moreover, previous studies confirmed that propofol has a hepatoprotective effect through its role in reduction of free radical generation and free radical scavenging, dumping of inflammatory reactions. [32,33] It has inhibitory effects on calcium channels and diminution of intracellular calcium overload, improved microcirculation through reported induced nitric oxide (NO) and vasodilatory prostanoid production. $[33,34]$

ROC curves revealed that miR-122 had the highest efficiency in prediction of ischemia/reperfusion injury followed by miR-223. These findings suggested that estimation of plasma miR-122 and miR-223 in CHC undertaking anesthesia could be an early, and reliable biomarker for predicting $\mathrm{I} / \mathrm{R}$ liver injury. Interestingly, these biomarkers were significantly related with $\mathrm{CHC}$ patients (SC and PC groups)with child-pugh score childpugh $\mathrm{B}$ than $\mathrm{A}$; thus these miRNAs could be considered as sensitive biomarkers for predicting liver stress and ischemia specifically in $\mathrm{CHC}$ patients receiving anesthesia.

Also, Sevoflurane is associated with increased risk of bleeding in $I / R$. As Sevoflurane produces prolonged bleeding times in normal patients, accordingly this effect is more exaggerated in $\mathrm{CHC}$ patients. The underlying mechanisms is thought to be through immediate inhibition of granulocyte-platelet aggregation by suppression of thromboxane A2 formation, inhibition of calcium mobilization in platelets and inhibition of platelet aggregation. [37,38,39]

Significantly reduced platelet count detected in our study predominantly in $\mathrm{CHC}$ groups. Concurring with other studies which reported reduced platelet counts, due to platelet accumulation in liver during I/R. [39,40,41] Kurokawa et al stated that platelets adhered to Kupffer cells at the early period of $\mathrm{I} / \mathrm{R}$ and that those platelets were involved in apoptosis of hepatocytes and subsequently stimulated platelet migration from sinusoidal space into the space of Disse. [42]

Significant reduction in MAP was observed in Sevoflurane anesthesia groups ( $\mathrm{S}$ and $\mathrm{SC}$ ) intra-operative and at the end of surgery may be the result of impaired auto-regulation. On the other hand, a more preserved hepatic function was perceived in groups receiving Propofol anesthesia ( $\mathrm{P}$ and PC). Although it is known that CHC patients might have alterations in the systemic circulation due to arteriovenous shunting and reduced splanchnic inflow, which makes liver more vulnerable to hypoxemia and hypotension during anesthesia. $[43,44]$

\section{Conclusion}

Our results suggested that both miR-122 and miR-223 could be considered more sensitive and specific predictors of hepatic $\mathrm{I} / \mathrm{R}$ injury than the traditional parameters of liver function (as ALT and AST) and serum lactate in CHC patients. Additionally, both sevoflurane and propofol anesthesia affect miRNA expression. However, further studies are required to determine the functional consequences of miRNA modulation in $\mathrm{I} / \mathrm{R}$ in $\mathrm{CHC}$ versus non-hepatitis patients.

\section{Decleration}

Acknowledgement: The authors are thankful to the science and technology development fund (STDF) for the financial support provided to accomplish this work.

Funding: This work was supported by a grant from the science and technology development fund (STDF), ministry of scientific research, Egypt, grant No. 15243.

Conflict of interest statement we declare that there is no disclose any financial and personal relationships with other people or organizations that could inappropriately Influence (bias) our work.

\section{References}

1. Ziser A, Plevak DJ, Wiesner RH, et al. Morbidity and mortality in cirrhotic patients undergoing anesthesia and surgery. Anesthesiology 1999; 90:42-53.

2. Banga NR, Homer-Vanniasinkam S, Graham A, Al-Mukhtar A, White SA, Prasad KR. Ischaemic preconditioning in transplantation and major resection of the liver. Br J Surg 2005; 92:528-38.

3. Zhai Y, Petrowsky H, Hong JC, Busuttil RW, KupiecWeglinski JW. Ischaemia-reperfusion injury in liver transplantation--from bench to bedside. Nat Rev Gastroenterol Hepatol. 2013;10(2):79-89.3.

4. Videla LA. Oxidative stress signaling underlying liver disease and hepatoprotective mechanisms. World J Hepatol. 2009;1(1):72-8.5.

5. Bahde R, Spiegel HU. Hepatic ischaemia-reperfusion injury from bench to bedside. Br J Surg. 2010;1461-75.56. 
6. Laterza OF, Lim L, Garrett-Engele PW, et al. Plasma microRNA as sensitive and specific biomarkers of tissue injury. Clin Chem 2009; 55:1977-1983.

7. Turchinovich A, Weiz L, Langheinz A and Burwinkel B: Characterization of extracellular circulating microRNA. Nucleic Acids Res 2011; 39: 7223-7233.

8. Wang K, Zhang S, Marzolf B, et al: Circulating microRNAs, potential biomarkers for drug-induced liver injury. Proc Natl Acad Sci USA 2009;106: 4402-07.

9. Ishak K, Baptista A, bianchi L, et al. Histological grading and staging of chronic hepatitis. J Hepatol 1995; 22: 696-99.

10. Stehling LC, Doherty DC, Faust RJ, Greenburg AG, Harrison CR, Landers DF, Laros RK, Pierce EC, Prust RS, Rosenberg AD, Weiskopf RB, Woolf SH, Zeiger JF. Practice Guidelines for Blood Component Therapy: A Report by the American Society of Anesthesiologists Task Force on Blood Component Therapy. Anesthesiology 1996; 84: 732-4.

11. Sakamoto A, Imai J, Nishikawa A, Honma R, Ito E, Yanagisawa Y, Kawamura M, Ogawa R, Watanabe S. Influence of inhalation anesthesia assessed by comprehensive gene expression profiling. Gene2005; 356:39-48.

12. Nakazato K, Yoshida Y, Takemori K, Kobayashi K, Sakamoto A. Expressions of genes encoding drug-metabolizing enzymes are altered after sevoflurane, isoflurane, propofol or dexmedetomidine anesthesia. Biomed Res 2009; 30:17-24.

13. Ishikawa M, Tanaka S, Arai M, Genda Y, Sakamoto A. Differences in microRNA Changes of Healthy Rat Liver between Sevoflurane and Propofol Anesthesia. Anesthesiology 2012; 117:1245-52.

14. Jaeschke H: Molecular mechanisms of hepatic ischemiareperfusion injury and preconditioning. Am J Physiol Gastrointest Liver Physiol 2003;284: G15-26.

15. Bihrer V, Friedrich-Rust M, Kronenberger B, Forestier N, Haupenthal J,

16. Shi Y, Peveling-Oberhag J, Radeke H, Sarrazin C, et al.Serum miR-122 as a biomarker of necroinflammation in patients with chronic hepatitis $\mathrm{C}$ virus infection. Am J Gastroenterol 2011; 106: 1663-69.

17. Van der Meer AJ, Farid WR, Sonneveld MJ, et al. Sensitive detection of hepatocellular injury in chronic hepatitis $\mathrm{C}$ patients with circulating hepatocyte-derived microRNA-122. J Viral Hepat. 2013; 20: 158-66.

18. Wang J, Jiang D, Rao HY, Zhao JM, Wang Y, Wei L. Absolute quantification of serum microRNA-122 and its correlation with liver inflammation grade and serum alanine aminotransferase in chronic hepatitis C patients. Int J Infect Dis 2015; 30: 52-56.

19. Subhi M, Islam M, Bassam AL, Mohamed M, Fayez S, Hamdi AA. Effects of sevoflurane on postoperative liver functions in morbidly obese as compared to the non-obese patients. M E J ANESTH 2009;20(2):207-11.

20. Choi Y, Dienes HP, Krawczynski K. Kinetics of miR-122 expression in the liver during acute HCV infection. PLoS ONE. 2013;8: e76501.
21. Pirola CJ, Fernandez Gianotti T, Castano GO, Mallardi P, San Martino J, Mora Gonzalez Lopez Ledesma M, et al. Circulating microRNA signature in non-alcoholic fatty liver disease: from serum non-coding RNAs to liver histology and disease pathogenesis. Gut 2015; 64:800-12.

22. Bala S, Petrasek J, Mundkur S, et al. Circulating microRNAs in exosomes indicate hepatocyte injury and inflammation in alcoholic, drug-induced and inflammatory liver diseases. Hepatology 2012; 56:1946-57.

23. Zhang Y, Jia Y, Zheng R, Guo Y, Wang Y. Plasma microRNA-122 as a biomarker for viral-, alcohol-, and chemical-related hepatic diseases. Clin Chem 2010; 56:1830-8.

24. Patrik A, Olof G, Oscar Ö B, Matthias G, Jesper V, Erlinge O. Plasma Levels of Liver-Specific miR-122 Is Massively Increased in a Porcine Cardiogenic Shock Model and Attenuated by Hypothermia. Shock 2012;37(2):234-38.

25. Ko J, Gwak M, Choi S, Yang M, Kim M, Lee J, Kim G, Kwon $\mathrm{C}$ and Joh J. The effects of desflurane and sevoflurane on hepatic and renal functions after right hepatectomy in living donors. European Society for Organ Transplantation 2010; 23: 736-744.

26. Koeppel TA, Thies JC, Schemmer P, Trauner M, Gebhard MM, Otto G, Post S. Inhibition of nitric oxide synthesis in ischemia/reperfusion of the rat liver is followed by impairment of hepatic microvascular blood flow. J Hepatol 1997; 27:163-69.

27. Yu C, Xu C, Li Y. Association of MicroRNA-223 Expression with Hepatic Ischemia/Reperfusion Injury in Mice. Dig Dis Sci 2009; 54:2362-2366.

28. Zhuang G, Meng C, Guo X, Cheruku PS, Shi L, Xu H, Li H, Wang G, Evans AR, Safe S, Wu C, Zhou B. A novel regulator of macrophage activation: miR-223 in obesityassociated adipose tissue inflammation. Circulation 2012; 125: 2892-903.

29. Takahashi K, Oda Y, Toyoda Y, Fukami T, Yokoi T, Nakajima M. Regulation of Cytochrome b5 Expression by miR-223 in Human Liver: Effects on Cytochrome P450 Activities. Pharm Res 2014; 31:780-94.

30. Nagai T, Egashira T and Yamanak Y. Effect of Bifemelane Hydrochloride on an Injury of the Liver Caused by IschemiaReperfusion in Rats. Japan J of Pharmacol 1990; 52: 383-385.

31. Qian YB, Liu ZG, Geng XP. Lack of protection of ischemic preconditioning in the rat model of major hepatectomy with ischemia reperfusion injury. Asian J Surg 2008; 31: 140-47.

32. Teoh NC, Farrell GC. Hepatic ischemia reperfusion injury: pathogenic mechanisms and basis for hepatoprotection. $\mathrm{J}$ Gastroenterol Hepatol 2003; 18: 891-902.

33. Lee J, Shin J, Lee E, Baek S, Ku S, and Kim J. Comparison of the effects of propofol and pentobarbital on hydrogen peroxide-stimulated hepatic SNU761 cells. Korean J of Anesthesiol 2010; 58(3):277-282. 
34. Vaja R, McNicol L, Sisley I. Anaesthesia for patients with liver disease. (CEACCP). 2009;10(1):15-9.

35. Xu Z, Yu J, Wu J, Qi F, Wang H, Wang Z, Wang Z. The Effects of Two Anesthetics, Propofol and Sevoflurane, on Liver Ischemia/Reperfusion Injury. Cell Physiol Biochem. 2016;38(4):1631-42.

36. Faga E, De Cento M, Giordanino C, Barletti C, Bruno M, Carucci P, et al. Safety of propofol in cirrhotic patients undergoing colonoscopy and endoscopic retrograde cholangiography: results of a prospective controlled study. Eur J Gastroenterol Hepatol. 2012;24(1):70-6.

37. Shimono H, Goromaru T, Kadota Y, Tsurumaru T, Kanmura Y. Propofol Displays No Protective Effect against Hypoxia/ Reoxygenation Injury in Rat Liver Slices Anesth Analg 2003;97:(2) 442-8.

38. Bozdogan N, Madenoglu H, Dogru K, et al. Effects of isoflurane, sevoflurane and desflurane on platelet function: a prospective, randomized, single blind, in vivo study Curr Therap Res 2005;66:375-84.

39. Yokubol B, Hirakata H, Nakamura K, et al. Anesthesia with sevoflurane, but not with isoflurane, prolongs bleeding time in humans. J Anesth 1999; 13:193-6.
40. Linden MD, Whittaker $\mathrm{P}$, Frelinger AL, Barnard MR, Michelson AD, Przyklenk K. Preconditioning ischemia attenuates molecular indices of platelet activationaggregation. J Thromb Haemost 2006; 4:2670-7.

41. Huang GS, Li CY, Hsu PC, Tsai CS, Lin TC, Wong CS. Sevoflurane anesthesia attenuates adenosine diphosphateinduced P-selectin expression and platelet-leukocyte conjugate formation. Anesth Analg 2004; 99:1121-6.

42. Pak S, Kondo T, Nakano Y,et al. Platelet adhesion in the sinusoid caused hepatic injury by neutrophils after hepatic ischemia reperfusion. Platelets 2010;21(4): 282-8.

43. Kurokawa T, Zheng Y, Ohkohchi N. Novel functions of platelets in the liver. J of Gastroenterol and Hepatol 2016;31: 745-751.

44. Montalvo-Jave EE, Escalante-Tattersfield T, Ortega-Salgado JA, Piña E, Geller DA. Factors in the pathophysiology of the liver ischemia-reperfusion injury.J. Surg. Res.2008;147(1): 153-9.

45. Suttner W, Schmidt C, Boldt J et al. Low flow desflurane and Sevoflurane anesthesia minimally affect hepatic integrity and function in elderly patients. Anesthesia Analg2000; 91(1):206-10.

*Corresponding author:

Hala Mourad Demerdash, Canal El Mahmoudia Street، Ezbet El-Nozha, Qism Sidi Gabir, Alexandria Governorate, Egypt

Phone: +20 33877000

Email: demerdashh@yahoo.com

Date of Submission : 15.10.2017

Date of Acceptance : 11.11.2017

Financial or other Competing Interests: None.

Date of Publication : 28.01.2018 